\title{
APUNTES PARA LA HISTORIA DE LA LEXICOGRAFÍA HISPANO-INGLESA*
}

LIDIO NIETO JIMÉNEZ / CSIC

Manuel Alvar EzQuerRa / UCM

Amado Alonso, en su reseña ${ }^{1}$ del Tesoro lexicográfico (1492-1726) de Samuel Gili Gaya, en una nota a pie de página, señalaba que en su largo trato con gramáticas antiguas se habia encontrado con algunos vocabularios menores incluidos en ellas, y que eran merecedores de un capitulo en la historia de la lexicografia, pues, pese a ser escuetos en unas ocasiones, otras veces discuten y comparan significados y traen noticias no usuales en los diccionarios. Entre esas gramáticas ponía The Key of the Spanish Tongve de Lewis Owen ${ }^{2}$. Amado Alonso se daba cuenta de la dificultad para incluir en una obra acumulativa como el Tesoro de Gili Gaya esos pequeños elencos, y escribía: "¿Cómo pedir aún más trabajo y más materiales a quien tanto y tantos ha derrochado y ha acopiado? Sólo como expresión de un inalcanzable ideal to podemos hacer" ${ }^{3}$.

Cuando nos enfrentamos con la redacción del Nuevo Tesoro lexicográfico de la Lengua Española (s. XIV-1726) (NTLE) tuvimos en cuenta esa advertencia y tratamos de seguir la indicación del maestro, aunque no siempre fue posible o conveniente, como explicaremos en el Prólogo del NTLE. En el caso concreto de Owen, al examinar detenidamente el contenido de su obra, llegamos a la conclusión de que no se justificaba su presencia ni en el Tesoro de don Samuel, ni en nuestro NTLE, por tratarse de un vocabulario monodireccional inglés-español, que, además, resultaba bastante pobre, teniendo en cuen-

\footnotetext{
" Este trabajo se encuadra dentro de Ios llevados a cabo para el proyecto "Nuevo Tesoro Lexicográfico del Español (s. xIV-1726)" que goza de una ayuda del Ministerio de Ciencia y Tecnologia (BFF2001-0263).

i $N R F H$, V-3, julio-septiembre 1951, págs. 324-328.

2 Lewis Owen, The Key of the Spanish Tongve, Londres, T. C. for W. Welby, 1605.

${ }^{3}$ Pág. 327 de la reseña citada.
}

RFE. LXXXIl, 2002, 3. ${ }^{\circ}-4^{\circ}$, págs. 319-343 
ta los antecedentes lexicográficos hispanoingleses en los que el español era la lengua de entrada. Bien es cierto que el segundo de los aspectos, esto es, la pequeña cuantía léxica, no hubiera sido obstáculo para su inclusión de haber sido el español la lengua de entrada. Hechas estas consideraciones, sí creemos que vale la pena tener en cuenta la obra de Owen en un panorama lexicográfico del español, razón que justifica las páginas que ofrecemos ahora.

The Key of the Spanish Tongve es un manual elemental para el aprendizaje del español, que consta de una primera parte, con contenidos gramaticales, seguida de cuatro diálogos, un pequeño diccionario alfabético (págs. 138-206), que acaba con la relación de los números y de los meses y estaciones del año, y una última parte que contiene textos de carácter doctrinal. El diccionario aparece bajo el encabezamiento de $A$ short Dictionarie, Alphabetically, or after the order of A.B.C., etc. Bien es verdad que el mencionado orden alfabético tiene numerosas transgresiones.

La relación de Owen añade más bien poco léxico español a los dos primeros repertorios bilingües conocidos ${ }^{4}$ y a los diccionarios español-inglés anteriores ${ }^{5}$, que nos servirán de punto de referencia. Hay que advertir que la obra de Owen no es una simple copia de ninguno de ellos. Registra nuestro autor una serie de voces que podemos considerar nuevas, como abstenit, accaer, acumplir, atapador, branar (que no es sino un error por bramar, voz ésta re-

\footnotetext{
${ }^{4}$ Son estos los anónimos sigutentes: $A$ very profitable boke to lerne the maner of redyng writyng \& speakyng english \& Spanish. Libro muy prouechoso para saber la manera de leen, y screuir, y hablar Angleis, y Español, Londres, John Kyngston and Henry Sutton for John Wyght, 1554; facsímil, Menston, The Scolar Press, 1971; y The Boke of Englysshe and Spanisshe, s. t. [Londres], Robert Wyer, s. a. [1554]; edición facsímil, Menston, The Scolar Press, 1971.

${ }^{5}$ La retación de estos diccionarios está encabezada por el contenido en la gramática de John Thorius, The Spanish Grammer: With certeine Rules teaching both the Spanih and French tongues [...] Made in Spanish, by M. Anthonie de Corro. With a Dictionarie adioyned vnto it, of all the Spanish wordes cited in this Booke: and other more wordes most necessarie for all such as de sire the knowledge of the same tongue, Londres, John Wolfe, 1590; edición facsimilar bajo el nombre de Antonio del Corro, The spanish grammar (1590), Menston, The Scolar Press, 1967.

Sigue a éste el de Richard Percyvall en la Bibliotheca Hispanica. Containing a Grammar. with a Dictionarie in Spanish, English, and Latine, gathered out of diters good Authors: very profitable for the studious of the Spanish toong, Londres, Jotn Jackson para Richard Watkins, 1591.

Con la misma fecha de Percyvall apareció el de William Stepney, The Spanish Schoolemaster. Containing seven Dialogues [...] Whereunto, besides seuen Dialogues, are annexed most fine Prouerbs and sentences, as also the Lords prayer, the Articles of our beliefe, the ten Commandements, and a Vocabularie, with diuers other things necessarie to be knowne in the said tongue, Londres, R. Field para John Harison, 1591.

Finalmente, tenemos en cuenta John Minsheu, A Dictionarie in Spanish and English, first published into the English tongue by Ric. Perciuale [...] Hereunto for the further profite and pleasure of the learner or delighted this tongue, is annexed an ample English Dictionarie [...], Londres, Edm. Bollifant, 1599; edición facsimilar, sólo del diccionario, con prólogo de Gloria Guerrero Ramos y Manuel F. Pérez Lagos, Málaga, Universidad de Málaga, 2000.
} 
cogida con anterioridad, pero no con el valor de 'regañar', que es el empleado por Owen), carisea (definida por el DRAE92 como 'tela basta de estopa, que se tejía en Inglaterra, muy usada en España en los siglos XVI y Xvlı para ropas de cama pobre'), christianidad (probablemente por influencia directa de la forma inglesa) en lugar de la voz christiandad, documentada en Minsheu con idéntico equivalente inglés. También encontramos cino, sinónimo de cisne (registrado igualmente por Owen), que puede ser un cultismo, no encontrado en los antecedentes manejados; dorso es voz nueva en Owen, traducción del término inglés backe, interpretado como espalda en $A$ very [...]; otras voces nuevas son enteçar y la probable forma anticuada fruente, que no aparece en los diccionarios considerados, aunque no es descartable que se trate de un simple error por frente, que sí se recoge antes; furruela, como sinónimo de manteo, tampoco la encontramos con anterioridad, si bien manteo aparece en Percyvall y Minsheu; más dificil es pronunciarse sobre fustana, que, de considerarla como adjetivo, sería voz nueva, y en el supuesto de interpretarla como error o variante de fustán si que tiene antecedentes con el mismo equivalente inglés; gescallera puede ser voz nueva o error por escalera, ya que el equivalente inglés, stilt, sí aparece al traducir zanco o ganco en otros autores, término éste con el que se podria relacionar la escalera; granaja, en cuanto traducción del inglés barn, es voz directamente emparentada con granera (Percyvall, Minsheu) y granero (A very $[. .$.$] , Stepney y Minsheu), aunque no nos parece$ un simple error; horologio, equivalente de relox, es, evidentemente, un cultismo no registrado con anterioridad; lealdad, voz que no vemos registrada antes en nuestros repertorios, es forma más antigua que la documentada lealtad, e igualmente podría pensarse del lymósina frente al limosna de $A$ very $[\ldots]$, Percyvall y Minsheu; linda, como equivalente de hermosa, tampoco aparece antes, aunque sí lindo; maçar es voz nueva, equivalente del inglés estampe, que Stepney y Minsheu traducen como majar, y el propio Minsheu y Percyvall como machucar, marir, no documentada antes, es sinónimo de casar, seguramente inducida por el inglés to marie; metar, equivalente de traer, y traducción ambas del inglés to leade, no vemos que pueda ser error de otra voz documentada con anterioridad y, por eso, a pesar de la dudosa razón de su existencia, la incluimos en este apartado; mosco, como voz aislada, no figura en los vocabularios anteriores, y parece un cultismo del latín muscus, pero consta en el compuesto gato de mosco (posiblemente el gato de algalia) que registran Stepney y Minsheu, en todos los casos con referencias al inglés mus$k e$; obrir y obrido pueden considerarse formas nuevas de carácter arcaizante; pierder, traducción del inglés to perish, no vemos que tenga relación con perecer, voz con la que Percyvall y Minsheu traducen el mismo término inglés; pimiento puede ser errata por pimienta $(A$ very [...], Stepney, Percyvall y Minsheu) o voz nueva, ya que ambas voces castellanas pueden traducir el 
pepper inglés al que se están refiriendo; quartero, sinónimo de quarto, no está documentado antes; regalemente, voz con la que Owen traduce el inglés kingly, que para Minsheu equivale a regio, tal vez sea error por realmente, si bien esta voz, documentada en Percyvall y Minsheu, tiene distintos equivalentes en inglés, really y roially, respectivamente; repossada, como sinónimo de cama, no aparece en repertorios anteriores; señar, traducción de to marke, es voz nueva, si bien no podemos olvidar que este mismo verbo inglés se interpreta por señalar en Percyvall y Minsheu; tábula puede ser error por tabla, voz ésta con la que Stepney traduce el mismo término inglés, board, o bien puede remitir directamente a la forma latina; tintar es palabra nueva, equivalente a teñir, término éste que utilizan los autores anteriores para traducir la misma expresión inglesa de Owen; vergüençar parece creación de Owen a partir de vergüença, que si se documenta en otros autores.

Cabe, además, señalar la presencia de algunos participios no registrados con anterioridad, si bien sí están los correspondientes infinitivos, como ocurre con almorsado (antes aparece almorçar y almorzar), procurado (se documenta procurar en Percyvall y Minsheu, así como en el propio Owen), prostrado (Minsheu y Owen documentan prostrar), respondido (responder aparece en $A$ very [...], Percyvall y Minsheu). También es baratillo una forma nueva, por más que sea un derivado de barato, pues el equivalente que proporciona Owen, good cheape, es el mismo que ponen Thorius, Percyvall y Minsheu para barato, forma ésta que también registra Owen, aunque con el solo equivalente cheape. En resumen, como decíamos al comienzo, no son muchas las voces españolas nuevas de Owen, aunque sí suficientes para no aceptar, sin más, la idea de plagio.

Otros casos, dentro de las diferencias con los repertorios anteriores considerados, se deben a variantes gráficas, que en algunas ocasiones se podrían interpretar como reflejo de variantes fonéticas, pues no debemos olvidar que Owen vivió en Castilla la Vieja unos años durante su etapa de jesuita ${ }^{6}$. Así, abroço frente al abrojo de Stepney, de Percyvall o de Minsheu (aunque en estos con otros equivalentes); accompañar frente a acompañar, que está en $A$ very [...], en Percyvall, y en Minsheu; aguasa se interpreta en inglés como waterish, que es el equivalente que pone Stepney para tierra aguosa; almorsar frente a almorçar en $A$ very [...] y en Minsheu, o almorzar en éste y en Percyvall; anguila frente a la más conservadora anguilla de Percyvall, Stepney y Minsheu; atreuido o atreuimiento que constan como atrevido y atrevimiento en Percyvall y Minsheu, si bien con otros equivalentes; auisar es avisar en estos mismos repertorios, aunque en ellos los equivalentes son distintos; $b a c ̧ i n$

${ }^{6}$ Véase Amado Alonso, De la pronunciación medieval a la moderna en español, I, 2. ${ }^{2}$ ed., Madrid, Gredos, 1967, pág. 209. 
aparecía como bacín en obras anteriores; bessar es besar en varios de los repertorios precedentes; bibir es bivir en Percyvall y Minsheu, y también vivir en el segundo; cappa coincide con la capa de Stepney y Minsheu; catiuo, caualgar, euitar, jugo, mançebo, nouiembre, oluidar, vaina, son cativo, cavalgar, evitar, yugo, mancebo, noviembre, olvidar, vayna, de los anteriores repertorios; colatión responde al colación o colaçión anteriores; comfortar es el confortar de repertorios precedentes, como enimigo lo es de enemigo, ruffián de rufián (o de rofián, varias veces documentado), probablemente por influencia de las correspondientes voces inglesas; algadón y cortez responden a algodón y cortés anteriores; deciembre es el deziembre de Percyvall (quien también registra diziembre) y Minsheu; delphin es el anterior delfin; despauillar es el despauilar de Percyvall y el despavilar de Minsheu, como destillar es el destilar anterior, y el moletas es el molletas de Stepney; desuariar, desuiarse corresponden a desvariar, desviarse; dizir es dezir en los precedentes; enbidioso es el embidioso de Percyvall y Minsheu; fuerza es la fuerça de los anteriores; huérphano aparece antes como huérfano y vérfano; impeyne e ingañar son el empeyne y el engañar de Stepney, Percyvall y Minsheu; infrenar es el enfrenar de $A$ very [...], Percyvall y Minsheu; inuierno y mill aparecen en otros lugares del repertorio de Owen como invierno y mil, formas documentadas antes; iuzgado es el juzgado de Percyvall y Minsheu; miserecordia y miserecordioso son los misericordia y misericordioso de Percyvall y Minsheu; pessadumbre equivale a pesadumbre en el $A$ very [...], Percyvall y Minsheu; pestilentia es la pestilencia en otros anteriores; sesenta por sessenta aparece en los demás elencos considerados; specias son las especias de Stepney y Minsheu, y la especia de Percyvall; stiércol se documenta como estiércol antes, si bien con distintas variantes en el equivalente inglés; teças en lugar de tejas, documentada en singular, puede deberse a error gráfico o a variante fonética, lo que nos permitiría insistir en lo indicado arriba respecto a la experiencia que tenía del habla nuestro autor; en este mismo caso se puede encuadrar el tussino por el tocino de Percyvall y Minsheu; turuas es diferente modo de escribir la turba con que Minsheu traduce turfe (en Owen turffes).

Hay un tercer grupo de palabras aparentemente nuevas en Owen que podríamos considerar como errores: açado por açadón que ya aparece en repertorios anteriores con el mismo equivalente inglés; aisir, que en $A$ very [...] figura como assir, y en Minsheu remite a asir, con distintas traducciones inglesas; apazignar es, sin duda, apaziguar, tal y como aparece en Percyvall y Minsheu; arana se documenta como araña en $A$ very [...], Percyvall, Stepney y Minsheu; aserter tiene un equivalente inglés que corresponde a aserrar, por lo que interpretamos que es una errata por aserrer; algo parecido sucede con assentir, cuyo equivalente corresponde a assentar en otros repertorios; azeyten tiene una $n$ de más, y como azeyte se recoge en Stepney, Percyvall y Mins- 
heu; benedizir es el bendezir de Percyvall y Minsheu, aunque posiblemente esté inducido por el benedicho de estos; bonidad es la bondad que hay en repertorios anteriores; broquiel aparece por el broquel registrado por Stepney, Minsheu y Percyvall, aunque en éste también es incorrecta la forma (borquel); Owen escribe como calcaña lo que en sus predecesores es calcañar, aunque podria interpretarse como calcaño, forma que también registra Minsheu para remitir a calcañar; calcecero parece un error por el calcetero registrado anteriormente; capillana probablemente responde a un error por capellán o capellanía que hemos encontrado documentados en los repertorios manejados; cartidor es error por curtidor, como cenir lo es por ceñir, cerraja por cerrojo, chequito por chiquito, cogujado por cogujada, colebra por culebra, coracol por caracol, currir por correr, emboracharse por emborracharse, empunçoñar por emponçoñar, escaper por escapar, escriuer por escrituir, establa por estable, estoruar por estorvar, estranar por estrañar, estreñar por estrenar, fortelacer por fortalecer, freyer por freyr, graña por grana, montana por montaña, otaño por otoño, pariento por pariente, peleja por pelleja, persequer por perseguir, pessademente por pesadamente, piscado y piscador por pescado y pescador, quexada por quixada, rábonos por rábanos, renir por reñir, sannar por sanar, saruoso por sarnoso, secudir por sacudir, temparano por temprano, tenebla por tiniebla, viergen por virgen, vina por viña; choca es la choça de Stepney y otros; cierruo es claramente erróneo, especialmente porque la palabra siguiente es cierua, documentadas ambas voces con anterioridad; crano es el cráneo de Stepney y de Minsheu; la cucharra es la cuchara de los anteriores, como despartar es el despertar de ellos.

En esta larga enumeración hay que seguir incluyendo derambadero por el derumbadero de Stepney, que corresponde al derrumbadero de Percyvall y Minsheu; flora, precedida de vna, para traducir a flower, que no es sino una mala concordancia; fuerto es el fuerte de los otros diccionaristas; gauilón, que también aparece como tal, aunque con grafia $v$, en Percyvall, es error por gauilán, documentado bajo esta forma en $A$ very [...], y como gavilán en Minsheu; halçón es errata por halcón como hedar por heder; hernero, traducción de ianuarie, lo interpretamos como error por enero (documentado antes), probablemente por influencia de hebrero que como tal aparece en $A$ very [...], Percyvall y Minsheu, aunque en Owen es febrero; ioyes es el joyas de Stepney; limpiosa es un error por limpiesa (limpieza); logero, mosquita, mulada son logrero, mosquito, muladar de Stepney, Percyvall y Minsheu; la forma maldita, traducción de accursed, nos inclinamos a interpretarla como error por maldito, ya que ésta es la forma con que Minsheu traduce el mismo término inglés, que en Te boke [...] y Percyvall es cursed solamente; mantela es el mantel de los anteriores vocabularios; maruillar es el marauillarse o maravillar(se) documentados antes; meol de nuez es el meollo de nuez de 
Stepney y Minsheu; ñule parece una errata por ñuve o ñtue, que se documentan respectivamente en Minsheu y Stepney, y que remiten a la misma voz inglesa que Owen; peignar es el peinar que vemos en $A$ very $[. .$.$] ; el piaça$ que aparece como sinónimo de pega, cuyo equivalente inglés es pie, es claramente error por picaça, documentado en Stepney, Percyvall y Minsheu; pinçaras parece error por pinças, voz con la que Stepney y Minsheu traducen el mismo término inglés, pincers; rumpar es error por romper, aunque no se puede olvidar que Minsheu también registra rumper; serenna del mar es un error por serena del mar en Stepney, y serena simplemente en Percyvall y Minsheu (no encontramos documentación alguna de sirena); tintoreo es un error por tintero, ya que ésta es la voz registrada por Stepney, Percyvall y Minsheu para traducir la misma voz inglesa; todar parece error por rodar, interpretamos torreyno como error por torrezno, voz documentada en varios autores, si bien el término inglés dado por Owen solamente aparece en $A$ very [...] con la traducción torrezno; truid paréce error por triud, voz con la que Stepney traduce el mismo treuet de Owen, y con la que Minsheu remite a trévede, y de ésta a treude, cuyo equivalente inglés es el mismo treuet.

Dentro de este apartado de errores hay algunos casos que podria pensarse que están condicionados por las formas inglesas que traducen. Asi, el augosto por el agosto de Percyvall y Minsheu, tal vez influido por la forma inglesa august, el carpentero de Owen se registra como carpintero en sus predecesores, en todos con remisión al inglés carpenter; deácono por el diácono de vocabularios precedentes, y fleagmático por flemático, es probable que estén influidos por las correspondientes formas inglesas; flute es error por el fluta de Stepney, traducciones ambas del inglés flute, interpretada ésta como flauta en Percyvall y Minsheu; otobre responde al otubre de $A$ very [...], Percyvall y Minsheu, probablemente debido al october inglés; persecutión es otro error inducido por la forma inglesa, en lugar de persecución; pictura es un error por pintura, debido a la forma inglesa, picture, que aparece tanto en Stepney como en Owen; lo mismo sucede con púplicamente, error de públicamente, pues el equivalente inglés, pupliquely, responde, a su vez, a un error, y a partir de ahí aparece el púplico, claro error por público; suffrir también es error por sufrir, seguramente influido por la forma inglesa suffer.

Más dificil resulta pronunciarse sobre palabras nuevas que son meras variantes formales de otros términos ya consignados, así alhombre, frente al alhombra de Stepney y de Minsheu o la alhambra de Percyvall, todas ellas con el equivalente inglés carpet; arguazil, que en varios de los repertorios anteriores es alguazil; baptizado, que en Minsheu es bautizado; çafrán consta como açafrán en varios repertorios anteriores, y como azafrán también en Minsheu; cedaça de sirgo aparece como cedaço de sirgo en Stepney; cenahoria se documenta en varios de los anteriores como çanahoria; clérico, 
frente al anteriormente documentado clérigo podría interpretarse como cultismo, o como error o variante formal; dispertar pudiera ser una forma anticuada en lugar del despertar de $A$ very [...], Percyvall y Minsheu; heredor cabe ser interpretado como cambio de sufijo frente al ya documentado heredero, o como error; sanctidad frente al santidad de Percyvall y Minsheu también se puede deber a influencia culta. En definitiva, resulta complicado, sin una investigación minuciosa de las diferentes voces, pronunciarse de manera concluyente sobre algunas divergencias.

Si pasamos ahora a una valoración global del repertorio de Owen con respecto a los que hemos considerado antecedentes, nos encontramos con que, desde un punto de vista numérico, las entradas recogidas por nuestro autor son notablemente inferiores a Percyvall y Minsheu, y muy semejantes a las de $A$ very [...], que a su vez supera a The boke [...] (el más reducido), y al repertorio de Thorius. De manera más concreta, podemos afirmar que algo más de un tercio de las entradas de The boke [...] coinciden con Owen, y que representan, en general, léxico de tipo común, excepción hecha de alguna voz como almendra o calderón, escasamente significativas. La no coincidencia se da en voces más específicas, no pocas de ellas relacionadas con el mundo rural, como comino, agraz, apero, auellana, etc., lo que nos lleva a una primera, y provisional, conclusión de independencia de ambos vocabularios o, si se quiere, de falta de influencia de The boke [...] en Owen.

En el caso de $A$ very [...] se produce un grado de coincidencia menor y, también, con léxico general, lo que no nos autoriza a pensar en un condicionamiento del primero respecto a Owen. De haberse producido éste, muchas de las voces que encontramos en $A$ very [...], y que pueden considerarse pertenecientes al léxico general, se habrían recogido por Owen.

El Dictionarie de Thorius coincide con Owen en torno a un $30 \%$ de las entradas del primero, mientras que el resto, en su mayor parte, se puede considerar léxico propio de Thorius, distinguiendo dentro de éste voces de carácter general, como pueden ser abogacia, acertar, adolecer, adorar, alimentar, y otras más específicas: afuziar, chapin, chamarra, chueca, etc., ninguna de las cuales son compartidas por Owen, lo que nos hace pensar que nuestro autor no se ha beneficiado del trabajo de Thorius.

Richard Percyvall supone un hito fundamental en la historia lexicográfica hispanoinglesa, ya que su obra es la primera verdaderamente extensa con las dos lenguas. Respecto a ella, el repertorio de Owen resulta muy reducido y, una vez hecho el contraste de las dos obras, nos encontramos con que, de nuevo, hay aproximadamente un tercio de palabras de Owen que no se localizan en Percyvall, aunque en bastantes casos son puras variantes formales de las que hemos dado cuenta más arriba. Entre estas voces, hay muchas que hoy consideraríamos generales, pero también las hay más espe- 
cíficas, como anguila, ansarina, baratillo, atapadura, carisea, etc., que nos pueden permitir pensar en otras fuentes, en el supuesto, dificil de precisar, de que Owen hubiera utilizado a Percyvall.

Coetáneo del elenco de Percyvall es el The Spanish Schoole-master de William Stepney, cuya extensión es muy cercana al repertorio de Owen. Contrastadas ambas obras, resulta que, aproximadamente, el $50 \%$ de ellas es coincidente, dándose la circuntancia de que entre las voces de Owen que no constan en Stepney existen muchas de carácter general, como abaxar, abril, acabar, alegre, alto, altura, etc., y, al mismo tiempo, entre las de Stepney que no aparecen en Owen, hallamos voces menos comunes, como alache, acelga, açote, açuçena, etc., todo lo cual nos movería a pensar que no hay entre ambos vocabularios influencia alguna.

Finalmente, nos encontramos con A Dictionary de John Minsheu, el más amplio de todos los considerados (téngase en cuenta que, prácticamente, duplica el número de entradas del de Percyvalil), respecto al que Owen sigue teniendo un número considerable de voces diferentes. Bien es cierto que, analizadas éstas, en la mayor parte de los casos, obedecen a errores o a diferencias gráficas, según ya vimos anteriormente, sin que ello excluya la presencia de algunas voces -las menos- propias. ¿Es posible, a la luz de esto, afirmar la dependencia de Owen respecto a Minsheu? Tal vez, pero no necesariamente, entre otras cosas porque, dado el reducido número de voces de Owen, y el amplio de Minsheu, es más que probable que, tratándose de palabras de carácter general, las del primero estén incluidas en las del segundo. Pero nos encontramos con no pocas divergencias, aunque sean meramente gráficas, que nos hacen dudar de una dependencia muy directa.

En definitiva, el léxico recogido por Lewis Owen en su The Key of the Spanish Tongve no es muy extenso, y, dados los numerosos repertorios anteriores, es muy posible que haya tenido en cuenta alguno de ellos, especialmente el de Minsheu, aunque sea dificil rastrear antecedentes concretos. Cabe, también, que el punto de partida no sea el español, como nosotros estamos considerando, sino el inglés, en cuyo caso se reforzaría la posibilidad de una influencia inmediata por parte de Minsheu, único que posee una parte alfabética, en sentido estricto, inglés-español.

Señalado lo que pueda tener de novedad el léxico español del repertorio de Owen con respecto a los vocabularios anteriores a que hemos hecho referencia, resta recopilar el contenido de este pequeño diccionario, anticipando los equivalentes españoles a las entradas inglesas, y reordenando alfabéticamente el léxico contenido, a partir de este cambio. Debemos advertir que, para la alfabetización de las entradas, hemos corregido aquelios errores que pueden dificultar más la consulta, si bien en el interior del artículo conservamos la forma original y damos, entre corchetes, la propuesta por nosotros. 


\section{VOCES ESPAÑOLAS DE OWEN}

abad, an abbot.

abadessa, an abbesse.

abanador, v, avandor.

abaxar, to bowe.

abaxo, below or beneath.

abeja, a bee.

abierto, v. obrido.

abraçar, to imbrace.

abril, april.

abrir, v, obrir.

abroço, abroços, thistles.

abrojo, v. abroço.

abstenir, to abstaine.

abuela, a grandmother.

abuelo, a grandfather.

acá, acá, aquí, here.

acabar, to ende; acabar, acumplir, to accomplish.

açadón, açado [açadón], a mattock.

accaer, to fall out.

accompañar, to accompanie.

accustumbrado, accustomed.

accustumbrar, to accustome.

açombre, a quart.

acontecer, to chaunce.

açotar, to smite.

açúcar, sugar.

açumbre, medio açumbre, pinta, a pint.

acumplir, v. acabar.

adelantar, to aduance or further.

adereçar, to garnish.

adónde, adónde váys?, where goe you?

agosto, $v$. augosto.

agua, water.

aguamanil, an ewer.

agudo, sharp.

águila, an eagle.

aguja, a needle.

agujeta, a point.

aguosa, aguasa [aguosa], waterish.

aguzar, to whet.

ajudar, to aide.

aiunar, to fast.

ajo, ajos, garlicke.

ala, $a$ wing.

alabarse, to boaste. alçado, lifted.

alçar, to lift $v p$.

alegre, glad.

alegría, gladnesse.

alférez, an enseigne bearer.

alfombra, v. alhombre.

algodón, algađón [algodón], bumhast.

alguazil, a sergeant.

alguno/a, alguno, some; alguno, any;

alguna vez, sometime.

alhombre, a carpet.

allá, yonder.

almendra, an almond; alméndraz [s],

almonds.

almidonar, to scratche.

almohada, a cushion; v. cabeçal.

almorsado, a breakefast.

almorsar, to breake fast.

alquilar, to hyre.

alto, high.

altura, highnesse.

alumbrar, to light.

ama, a nurse.

amado, loued.

amar, to loue.

amarganente, bitterly.

amargo, bitter.

amargura, bitternesse.

amarillo, yellow.

amassador, a baker.

amassar, to bake.

ámbar, an amber stone.

amblar, to amble.

amigablemente, friendly.

amigo, a friend.

amistad, friendship.

amonestado, exhorted.

amonestar, to exhort.

anchura, breadth.

ancla, an anker.

ángel, an angell; ángeles, angells.

anguila, an ecle.

ánima, the soule.

ánsar, ansares, gansos, geese.

ansarina, a gosselin.

antojo, antojos, spectacles. 
año, a yeare; medio año, halfe a yeare; cada año, yearely.

aparador, a cupbord.

aparejado, prepared.

aparejar, to prepare.

apaziguar, apazignar [apaziguar], to content.

apocar, to diminish.

apostar, to wage.

apóstol, apóstolos, the apostles.

apretar, apretar, ligar, to bind.

aquel/ella, aquel, él, he; aquella, her.

aquí, aquí está, he is here; v. acá.

arado, a plough.

arador, a ploughman.

araña, arana [araña], a spider.

arar, to plough.

archero, an archer.

arco, arco del cielo, a rainebowe; v. arçón.

arçón, arçón, arco, a bowe.

arena, sand.

arguazil, $a$ baily or seriant.

arma, armour.

armado, armed.

armar, warme.

arribar, arriuabar [arribar], to arriue.

arrojar, ar\{rlojar, to cast.

arte, v. grammática.

aserter, to sawe.

asno, v. borrico.

assadero, a spit.

assado, a broach.

assegurar, to assure.

assentir, to sit.

assí, also.

assir, aisir [assir], to gripe.

astrología, astronomie.

astrólogo, an astronomer.

atapador, a stopple.

atapar, to stop.

atreuido, stoute.

atreuimiento, stoutnesse.

auaricia, couetousnesse.

auariento, couetous.

auena, oates.

auer, to haue.

augosto, august.

auisar, auisar, pensar, to bethinke. aullar, to yell, or crie out.

aún, yet.

avandor, v. vieldo.

aventar, to blowe like the wind.

ayer, yester day; ayer tarde, yester day

in the euening.

ayrado, angrie.

ayrarse, to be angrie.

ayre, the aire.

ayuntar, v. recoger.

azeyte, azeyten [azeyte], oile.

azeytuna, an oline.

azeytuno, an oliue tree.

azogue, quick siluer.

azul, blewe.

azumbre, v. açombre.

baçín, a bason.

bala, $a$ bullet.

balança, a ballance.

ballesta, a crosse bowe.

ballestero, a crosse-bow-maker.

bañar, to bath.

baño, $a$ bath.

baptizado, baptised.

baptizar, to baptise.

baratillo, good cheape.

barato, cheape.

barba, the chinne; a beard.

barbero, a barber.

barril, bar[r]il, a barrell.

batalla, ba[ta]lla, a battell.

batanar, to be ate.

baylar, to daunce.

becerro, v. bizerro.

bellota, an acorne; bellotas, acornes.

benedizir, to blesse.

berça, berças, cabages.

bessar, to kisse.

bestia, a beast.

bestialidad, beastlinesse.

beuer, to drinke.

bexiga, the bladder.

bibido, bibido, bibió, liued.

bibir, to liue.

bien, well; v. bueno.

bisabuelo, greet grandfather.

biuda, $a$ widow.

bizerro, a calf. 
blanca, $a$ bench.

blanco, white.

blanquear, to whyten.

boca, a mouth.

bocado, bocad[o], a bit.

bola, a bowle.

bolar, to flee.

bolsa, a purse.

boluer, to returne; to giue againe; boluendo, returning.

boneta, v. gorra.

bonidad, goodnesse.

boquear, to gape.

bordón, a staffe.

borracho, bor[r]acho, a drunckard.

borrico, borrico, vn asno, an asse.

botilla, a bottle.

botón, a button.

braço, an arme.

braga, bragas, a pair of breeches.

bragueta, a codpeece.

branar, to grinne.

bronze, brasse.

broquiel, a buckler.

brotar, to budde.

buen, muy buen, very good.

bueno, bueno, bien, good.

buey, an oxe; bueys, oxen.

buscar, to seeke.

cabeça, the head.

cabeçal, cabeçal, almohada, a pillow.

cabello, cabellos, the haires.

cabra, a she goate.

cabrito, a kid.

cabrón, a he goate.

caçador, a hunter; caçador de aues, a fowler.

caçar, to hunt.

cada, euery; cada muger, euery woman. cadena, a chaine.

caer, to fall; caer en pena, to forfeit.

çafir, a saphyr stone.

çafrán, saffron.

cagado, be shitten.

caherir, to cast in ones teeth.

calauaça, a pumpion.

calcaña, the heele.

calcetero, calcecero [calcetero], a hosier. calçón, calçones, sockes.

calderero, a tinker.

calderón, a kettle.

calentar, to warme.

calentura, the ague.

cáliz, a challice.

callar, to say nothing.

calle, a streete.

callexuela, a lane.

calxón, calzones, a paire of hose.

cama, cama, repossada, $a$ bed.

cámara, a chamber.

cambiar, v. trocar.

camello, a cammell.

camino, the way.

camisa, a shirt.

campana, $a$ bell.

campanero, a bellmaker.

camueça, a pippin.

cana, a frogge.

canastillo, a basket.

çanco, a crutch; lame.

candela, candela, vela, a candle.

candelero, a candlesticke; $a$ chandeler.

canela, sinamon.

canónigo, a chanon.

cantar, to sing.

cantero, a bricklayer.

cantón, a corner.

cantor, a singer.

çapatero, a shoe maker.

çapato, çapatos, shooes.

capilla, $a$ chappell.

capillana, a chapplaine.

capitán, a captaine.

capítulo, a chapter.

capón, a capon.

cappa, a cloake with a hood.

cara, v. rostro.

caracol, coracol [caracol], cockles.

carbón, carbones, coales.

cárcel, prison; a gaole.

carcelero, a gaoler.

cardinal, a cardinal.

carga, a burthen.

carisea, carsie.

carne, flesh; carne de puerco, porke; carne de vacca, beefe; carne de sierua, venison. 
carnecero, a butcher.

carnero, a wether; carnero cojudo, a ramme.

carnero, ca[r]nero, mutton.

carnicería, fleash house.

caro, deare.

carpentero, a carpenter.

carpio, a carpe.

carretero, a carter; a wagoner.

carro, a cart.

carta, cartas, naipes, cardes to play; v. letra.

casa, a house.

casar, casar, marir, to marie.

castaña, a chesse-nut.

castigar, to chasten.

castilla, a castle.

catiuo, a prisoner.

catorze, fourteene.

cauador, a ditcher.

caualgar, to ryde.

cauallero, a gentleman; a knight.

cauallo, $a$ horse; cauallo sin cola, a curtall.

cauar, to digge.

cauerna, a denne.

caxero, aioyner.

cebolla, an onion.

cedaça, cedaça de sirgo, a boulter.

ceja, cejas, the eye lids.

celada, a helmet.

celebro, the braine.

celemín, a bushell.

cena, a supper.

cenahoria, cenahorias, carrotes.

cenar, to supp.

ceñir, cenir [ceñir], to gird.

cera, waxe.

cerca, neare.

cercar, to be seege.

cereza, cerezas, cherries.

cerezo, a cherrie tree.

cernir, cernir harina, to boult meale.

cerrada, cer[r]ada, locked.

cerrar, cer[r]ar, to locke.

cerrar, to shut, or locke.

cerroja, cer[r]oja, a lock.

chequito, a little one.

chiminea, a chimney. chiribia, chiribias, parsneps.

choca, a farme.

chocarrero, a ingler.

christianidad, christianitie.

christiano, christian.

cielo, heauen.

ciento, hundred; one hundreth.

cieruo, cierruo [cieruo], a hart.

cierva, a hinde.

ciervo, v. cierruo.

cigarra, a grashopper.

cincha, a girt.

cinco, fiue.

cino, cino, cisne, a swanne.

cinquenta, fiftie.

ciruela, a plumme.

ciruelo, a plumme tree.

cisne, v. cino.

ciudad, a citie.

ciudadano, a cittizen.

claridad, brightnesse.

clauellina, a gilliflower.

clauo, a naile; clauos, cloues.

clérico, $v$, sacerdote.

cobertor, a couerlet.

cocina, v. cuzina.

codo, the elbow.

codorniz, a quaile.

cogujado, a larke.

cojón, cojones, the cods.

cojudo, v. carnero cojudo.

cola, a taile.

colar, to wring.

colatión, a colation.

colebra, an adder.

colgar, to hang.

color, a colour.

colorado, coloured.

comadre, a shee gossip.

començar, to begin.

comer, to eate; to dine.

cometa, a comet.

comezón, itch; tener comezón, to itch.

comfete, comfetes, comfets.

comfortar, to comfort.

comida, a dinner.

cómo, how.

compadre, a gossip.

compañero, acompanion. 
compás, a compasse.

competir, to cleaue.

comprar, to buy.

común, common.

concebir, to conceitue or bee with childe.

conciertar, conciertar de precio, to

bargaine.

conde, an earle.

condemnar, to condemne.

condessa, a countesse.

conejo, a cunnie.

confessar, to confesse.

confessión, confession.

congrejo, a crabbe.

congrio, a conger.

conocer, v. saber.

consagrar, to hallow.

consejar, to counsell.

consejero, a counsellour.

consejo, counsel.

consentir, to consent.

consolado, comforted.

consolar, to comfort.

constancia, constantnesse.

constante, constant.

constreñitir, to constreine.

contante, contantes, counters.

contar, to tell; to recken.

contra, against.

coraçón, a heart.

cordero, a lambe.

corredor, a broker.

corregir, to correct.

corta, short.

cortador, cortador de bolsas, a cuttepurse.

cortar, to cut.

cortés, cortes [cortés], v. franco.

corteza, cortezas, parings.

cosedura, a seamster.

coser, to sowe.

costal, a sacke.

costar, to cost.

costilla, a ribbe.

coxo, a cripple.

cozedor, cozedor de cerueza, a bruer.

cozer, to seeth; cozer cerueza, to brewe beare.

cozinero, a cooke. cráneo, crano [cráneo], the braine pan.

creer, to beleeue.

crónica, a chronicle.

cuba, $a t u b$.

cuchara, cucharra [cuchara], a ladell.

cuchillo, a knife.

cuello, the necke.

cuerda, a line, or corde; a cord.

cuero, leather.

cuerpo, a body.

cueruo, a rauen.

cuesta, a hillock.

cueua, a celler.

cuidado, care; tener cuidado, to care.

cuidadoso, careful.

culo, an arse.

cumplir, to fulfill.

cuna, a cradle.

cuña, $a$ wedge.

cunado, a brother in law.

currir, to runne.

curtidor, cartidor [curtidor] de cuero, $a$ tanner.

curtina, a curtaine.

cuxina, a kitchin.

dado, dados, dice to play.

dama, a gentlewoman.

dañar, to do amisse; to damnifie.

dar, to gine.

deácono, a deacon.

deciembre, december.

declarar, to declare.

dedal, a thimble.

dedo, dedos de los pies, toes; dado [dedo], a finger.

defender, to defend.

degollar, to behead.

delante, before.

delgado, slender.

delphín, a dolphin.

dende, $v$, desde.

derecho, d[e]recho, right.

derramar, to sheade.

derritido, molted.

derritir, to melt.

derrumbadero, derambadero [derrumbadero], a steepe hill.

desamparar, desampar[ar], to forsake. 
desde, desde, dende, since.

desheredar, to disherite.

despauillar, to snuffe a candle.

despertar, despartar [despertar], to waken.

desplegar, to unfold.

despojar, to strippe one.

después, after.

dessear, to desire.

desseo, desire.

destillar, to droppe.

destruyr, to destroy.

desuariar, to dote.

desuiarse, to goe astray.

dexar, to leaue.

día, a day; cada día, euery day; cada

día, daily; quinze dias, a fortnight.

diablo, the diuell.

diecinueve, diez y nueve, nineteene.

dieciocho, diez y ocho, eighteene.

dieciséis, diez y seys, sixteene.

diecisiete, diez y siete, seauenteene.

diente, dientes, teeth.

diez, tenne.

diligente, diligent.

dinero, dineros, v. moneda.

dios, god.

dispertar, dispertar del sueño, to awa-

ke out of sleepe.

dizir, to say.

domar, to tame.

doméstico, tame.

domingo, sonday.

domingo, sunday.

donde, whence.

donzella, v. moça.

dorar, to gild.

dormir, to sleepe.

dorso, the backe.

dos, two.

doze, twelue.

dulce, sweete.

dulcemente, sweetly.

duque, a duke.

duquesa, $a$ dutchesse.

dureza, hardnesse.

duro, hard.

echar, to fill.

edad, age. edificar, to build.

él, v. aquel.

elella, el, the; ella, shee.

elar, to freez.

embiar, to send.

embidia, enuie.

emborracharse, embor[r]acharse, to be drunken.

embudo, a tunnell.

emendar, to amend.

emperador, an emperour.

emperatriz, an emperesse.

empero, yet not withstanding.

empeyne, impeyne [empeyne], the instep.

emplear, to employ.

empreñarse, to be with childe.

emprestado, borrowed.

emprestar, to borrow; to lend.

empunçoñar, to poyson.

enbidia, tener enbidia, to enuie.

enbidioso, enuious.

encantar, to bewitch.

encender, to kindle.

encienso, frankencense.

encubierto, close.

encubrir, to close.

enero, hernero [enero], ianuarie.

enero, v. hernero.

enfermar, to sicken.

enfermedad, sickenesse.

enfermo, a sick man.

enfrenar, infrenar [enfrenar], to bridle. engañar, ingañar [engañar], to deceiue.

engendrar, to beget.

enimigo, an enimie.

ensalada, a salet.

enteçar, to cup vp.

entena, the forehead of a ship.

enterrado, enter[r]ado, buried.

enterrar, to burie.

entraña, entrañas, the entrailes.

entrar, to enter.

entregar, $v$ intregar.

enzía, enzías, the gummes.

errar, to erre.

escaper, to escape.

escarnecedor, a cogger.

escarpin, escarpines, pumpes.

escoba, $a$ brush; a broome; a rubber. 
escobero, a broome man.

escojer, to choose.

esconder, to hide.

escriuer, to write.

escuchar, to hearken.

escudilla, $a$ dyshe.

escudo, a crowne; a shield.

escuela, a schoole.

escupir, v. vomitar.

escurecer, to darken.

espada, a sworde; a rapier.

espadero, a cutler.

espalda, espaldas, the shoulders.

espantar, to feare.

español, a spaniard.

especia, specias [especias], spices.

espejo, a looking-glasse.

esperar, to hope; to tarrie; to waite.

espia, espias, spies.

espiar, to espie; to spie.

espiga, a blossome.

espinaço, the backe bone.

esposo/a, esposo, a bridegroome; espo-

sa, $a$ bride.

espumar, to scumme.

espumero, a scummer.

esquilo, a squerrell.

estable, establa [estable], a stable.

estaīero, a pewterer.

estaño, pewter; tynne.

estender, to stretche; to bende.

estiércol, stiércol [etiércol], dungue.

estimar, to esteeme.

estómago, the stomack.

estoruar, to hinder.

estraîar, estranar [estrañar], to be estranged.

estrella, a starre.

estrenar, estreñar [estraenar], to giue earnest.

euitar, to auoyde.

fagota, a fagot

faisán, a pheasant.

falta, a fault; want.

faltar, to want; to lacke.

fe, faith.

febrero, februarie.

felicidad, felicitie. feno, v, heno.

fiador, a suretie.

fiar, to credit.

fiel, v. leal.

filo, an edge.

firmamento, the firmament.

flauta, flute [flauta], a flute.

flautador, a fifer.

fleagmático, fuli of fleagme.

flecha, an arrow.

flora, a flower.

florecer, to blossome.

floretada, a fillip.

fluxo, fluxo del vientre, the squirt.

fontenal, v. fuente.

forçar, to force.

forjar, forjar dinero, to coine.

forma, a forme.

fortalecer, fortelacer [fortalecer], to fortifie.

fortaleza, a bulwark.

fortuna, fortune.

fraile, a fryer.

francés, a frenchman.

franco, franco, cortez, courteous.

fregar, to rubbe.

freír, freyer [freír], to frie.

freir, to frie.

freno, a bridle.

fresno, an anstree.

frío, colde.

fruente, a forehead.

fruta, frute.

fuego, fire.

fuelte, fuelles, a pair of bellowes.

fuente, fuente, fontenal, a fountaine; fuente, pozo, a well.

fuerte, fuerto [fuerte], strong.

fuerza, strength.

furruela, furruela, manteo, a cloake.

fustana, fustian.

galápago, a snaile.

gallina, gal[l]ina, a henne.

gallo, a cock.

ganar, to winne.

ganso, a goose; gansos, v. ánsar.

garauato, g[a]rauato, a pot hooke.

garguero, the throate. 
gastar, to dispend.

gato, a cat.

gauilán, gauilón [gauilán], a sparrowhawke.

género, a kind.

gente, folkes.

gescallera, a stilt.

gobio, gobi[o], a gudgion.

golondrina, a swallow.

golosina, delicatenesse.

goloso, delicate.

golozina, golozina, gula, gluttonie.

gomitar, gomitar, vomitar, to vomite.

gordura, fat.

gorra, gorra, boneta, a cap.

gota, a drop.

gotar, to drop.

gouernar, to rule.

grado, a stayer.

grammática, grammática, arte, a gram-

mar.

gran, gran, grande, greate.

grana, graña [grana], corne.

granada, a pome granate.

granaja, a barn.

grande, v. gran.

grandeza, greatnesse.

gritar, to crie out.

gruesso, humbre gruesso, a grosse man. grulla, a crane.

guante, guantes, gloues.

guardar, to keepe; to saue.

gula, v. golozina.

gusano, a worme.

gustar, to bestowe.

hablar, to speake.

bacha, a hatchet; a torch.

halcón, halçón [halcón], a faulcon.

hallado, found.

hallar, to finde.

hambre, hunger.

harenca, a hereing.

harina, meale.

harpa, a harpe.

hazer, to doo; to make.

hecho, don; made.

heder, hedar [heder], to stinck.

hender, to cleaue. heno, heno, feno, hay.

heredor, an heire.

hermano/a, hermano, a brother; hermana, a sister.

hermosa, v. linda.

hermosamente, fairely.

herrada, a buclet.

herrero, a smith.

hierro, yron.

hígado, the liuer.

higo, a figge.

higuera, a fig tree.

hijastro/a, hijastro, a god sonne; hijastra, a god daughter.

hijo/a, hijo, a sonne; hija, a daughter.

hilar, to spin.

hilo, hilo de lana, yarne.

hinchar, to swell.

hinojo, hin[oljo, fennel.

hoce, a sickle or syeth.

hojaldre, a custard.

bojo, a leafe.

hombre, a man.

hondo, deepe.

hondura, deepnesse.

honrado, honourable.

honrar, to honour.

hora, an howre; a qué hora, at what a clocke.

horadar, to bore.

horno, an oven.

horologio, v. relox.

hortiga, a nettle.

hoyo, a ditch.

hueco, hollowe.

huérphano, an orphant.

huésped/eda, huésped, an host; huéspeda, an hostesse.

huesso, a bone.

hueuo, an egge.

humillar, to humble.

humo, smoke; hazer humo, to smoke.

hundir, to sinke.

huso, a spindle.

huyr, to flie from or aduoid.

hypócrita, an hypocrite.

ídol, an idoll.

imagen, an image. 
impressión, the print.

impressor, impressores, printers. imprimir, to print.

inclinar, to make courtesie.

infierno, hell.

inglés, ingleses, englishmen.

intregar, to deliuer.

inuierno, winter; $\mathrm{v}$, invierno.

inuocar, to call vpon.

invierno, the winter; $\mathrm{v}$. inuierno.

ioya, ioyes [ioyas], iewels.

ira, anger.

irlandés, irlandeses, irishmen.

isopo, hysope.

iudío, a iewe.

iuez, $a$ iudgs.

iulio, iuly.

iunio, iune.

iusto, iust

iuzgado, itdged.

iuzgar, to iudge.

jardín, a garden.

jardinero, a gardiner.

jarro, a pot.

jauali, a wild boare.

jaula, a cage.

jubón, a doublet.

jueues, thursday.

jugar, to play.

jugo, a yoke.

juntar, juntar sílabas, to spell.

jurado, sweare.

jurar, to sweare.

justicia, iustice.

labio, labios, the lippes.

labor, labor, trabajo, labour.

ladrar, to bark.

ladrillo, a quaell of stone.

ladron, $a$ theefe.

lana, wooll.

lanterna, a lantern.

largo, long.

lauar, to wash.

leal, leal, fiel, faithfull.

lealdad, faithfulnesse.

leche, milke; leche de manteca, butter milke. lechón, a pigg.

lechuga, lechugas, lettice.

lengua, the tongue.

leîia, woode.

letra, letra, carta, a letter.

leuadura, leauen.

ley, the lawe.

libra, $a$ pound.

libre, free.

libremiento, freedome.

libro, a booke.

lienço, linnen cloth.

liga, ligas, garters.

ligar, v. apretar.

limar, to daube.

limpiamente, cleanly.

limpieza, limpiosa [limpieza], clealinesse.

limpio, cleane.

linage, v. pariento.

linda, linda, hermosa, faire.

llamar, to call.

lleno, full.

lleuar, to beare or cary.

llorar, to weepe.

llouer, to raine.

lobo, a wolfe.

locamente, foolishly.

loco, a foole.

locura, foolishnesse.

lodo, durt.

lodoso, durtie.

lógica, logick.

logrero, logero [logrero], an vsurer.

logro, vsurie.

luego, by and by.

lugar, a place.

lumbre, v, luz.

luna, the moone.

lunes, munday.

luz, luz, lumbre, light.

lymósina, almes.

maçar, to stampe.

madrastra, mad[r]astra, a mother in lawe.

madre, a mother.

madrina, a god mother.

maestro, a maister.

magro, leane. 
mal, euill; naught. maldezir, to curse. maldita, accursed. mançana, an apple. mançano, an appletree. mançebo, v. moço. manchado, spoted. manchar, to defile; to spot. mandar, to commaund. manera, a fashion. mano, $a$ hand. manojo, a sheaff. manso, meate. manteca, butter. mantel, mantela [mantel], a cloath for the table.

manteo, v. furruela.

maña, maña barreada, a barre of yron. mañana, the morning.

marauillar, mar[a]uillar, to wonder.

março, march.

marido, a husband.

marinero, a mariner; a shipper.

mariposa, a butterflie.

marir, v. casar.

martes, tuesday.

martillo, a hammer.

mas, but.

más, more.

mascar, to gnaw.

matar, to kill.

maxilla, maxillas, the cheekes.

mayo, may.

mayor, a maior.

media, medias, stockins.

medio, halfe.

mediodía, a medio día, at noone.

medir, to cure.

mejor, the beast.

melón, a cowcumber.

membrillo, a quince aple.

menor, yongest.

menospreciar, to despise; to contemne.

menosprecio, contempt.

mentir, to lye.

meollo, meol[lo] de nuez, a kernell of

a nut.

mercader, a manchaunt.

mes, a moneth; v. mez. mesa, a table.

mesmo, selfe.

metal, mettle.

metar, v. traer.

mez, a moneth; v, mes.

mi, mi, mio, mía, my.

miel, honnie.

miembro, a member.

miércoles, wednesday; wensday.

mierda, $\mathrm{m}[\mathrm{i}] \mathrm{erda}$, a toord.

miesse, haruest.

milano, a kite.

mill, one thousand; diez mil, ten thotsand; cien mil, an hundred thousand.

millión, a million; diez million[e]s, ten millions; cien miliones, an $100 \mathrm{mi}$ llions; mill milliones, a 1000 millions.

minar, to digge.

mío/a, v. mi.

mirar, to looke.

miserecordia, pittie.

miserecordioso, pittifull.

moça, moça, donzella, a maide.

mochacho, a boy.

moço, moço, mançebo, a young man.

moler, to grind.

molinero, a miller.

molino, a mill.

molletas, moletas [molletas], snuffers.

moneda, moneda, dineros, money.

monedero, a coiner.

monge, a monke.

mono, an ape.

montaña, montana [montaña], monte, $a$ mountaine.

monte, v. montana.

montón, a heape.

mora, a mulber[r]ie; mora de montaña, a strawber[r]ie.

moral, a mulber[r]ie.

morar, to dwell.

morçilla, a pudding.

morder, to bite.

morir, to dye.

moro, moros, barbarianes.

mosca, a flie.

moscada, a nutmegge.

mosco, muske.

mosquito, mosquita [mosquito], a gnat. 
mucho, mucho, mulo, mula, a mule. mudable, changeable.

mudo, dumbe.

muerto, death; los muertos, the dead. muger, a woman.

mula, v. mucho.

muladar, mulada[r], a dunghill.

mulo, v. mucho.

muro, $a$ wall of a towne; muros, walles.

música, musick.

musquete, a musket.

musquetero, a musketier.

nabo, nabos, turnips.

nacer, to beare.

nacido, born.

nada, nothing.

nadar, to swimme.

naipe, naipes, $v$. cartas.

nalga, nalgas, the buttockes.

naranja, an orenge.

nariz, the nose; narizes, the nostrels.

nata, creame.

nauaja, a razor.

nauigar, to sayle.

nauío, a shipp.

necessidad, necessitie.

negro, black.

nido, $a$ nest.

nieue, snowe.

ninguno, ninguno hombre, no man.

niño, a babe or childe.

no, no; no señor, no syr.

noche, the night.

nombre, a name.

nosotros, vs; wee.

nouenta, ninetie.

nouiembre, nouember.

nudo, a knot; nudo de la mano, a

knackl of a hand.

nuera, a daughter in law.

nuewo, newe.

nueve, nine.

nuez, a nut.

ñube, ñule [ñube], $a$ cloude.

obedecer, to obaie.

obispo, a bishop. obra, worke.

obrar, to worke.

obrero, a workeman.

obrido, opened.

obrir, to open.

ochenta, eightie.

ocho, eight.

octubre, v. otobre.

odre, a vessel.

ofensa, an offence.

offrecer, to offer.

offrenda, offrendas, offrings.

ojo, an eye; ojos, eyes.

oluidar, to forget.

ombligo, the natill.

omiziano, a murderer.

onze, eleuen.

orar, to pray.

oreja, an eare.

orinal, an vrinal.

oro, gold.

otobre, october.

otoño, otaño [otoño], the autumme.

oueja, a sheepe; v. oveja.

oveja, a yeaw; an eawe; v. oueja.

oýdo, the hearing.

oydor, an auditor.

oýr, to heare.

padastro, a father in law.

padecer, v. suffrir.

padre, a father.

padrino, a godfather.

pagar, to pay.

paja, straw.

pala, a shouell.

paladar, the roofe of the mouth.

paloma, a doue.

pan, bread; pan de centeno, bread of

rye; pan de ceuada, barly bread.

paño, wollen cloath.

papa, the pope.

papel, paper.

pardo, gray.

parecer, to appeare.

pared, the wall of a house.

pariente, pariento [pariente], linage, kinred.

parir, to beare children.

partera, a midwife. 
partido, parted.

partir, to part; to diuide.

pascua, easter.

passado, passed.

passamano, a lace.

passar, to passe.

passear, to walke.

pastel, a pastie.

pasto, fodder.

pastor/ora, a sheapheard.

pato, a ducke.

pauón, a peacocke.

páxaro, a bird.

paz, peace.

pecado, sinne.

pecar, to sinne.

pecho, the breast.

pedo, a fart.

peer, to fart.

pega, pega, pi[c]aça, a pie.

peinar, peignar [peine], to combe.

peine, a comb.

pelear, to fight.

pelleja, peleja [pelleja], a skinne.

pena, paine.

penar, to byde.

péndola, a pensill.

pensar, v. auisar.

peojo, a lowse; lleno de peojos, full of

lice.

pequeño, pequeño, poco, little.

pera, a peare.

perder, to loose.

perdido, lost.

perdiz, a partrich.

perdonar, to pardon; to forgiue.

perdurable, euerlasting.

perfumar, to be-smoke.

perla, a pearle.

pero, pero, por tanto, therefore.

perra, a bitch.

perro, a dogge; perro de rastro, a blood hound.

persecutión, persecution.

perseguir, persequer [perseguir], to persecute.

pertenecer, pertenecer a, to appertaine at. pesado, heauie.

pesar, to weigh; to greeue. pescar, to fish.

pesebre, a manger.

peso, weight.

pessadamente, pessademente [pessadamente], heauily.

pessadumbre, heauinesse.

pestilentia, the pestilence.

pez, pitch.

philosophía, philosop[h]ía, philosophie.

philósopho, a philosopher.

picaça, v pega.

pictura, a picture.

pie, a foote; banco del pie, a foote-stoole.

piedra, a stone.

pierder, to perish.

pierna, a legge.

piloto, a pilote.

pimiento, pepper.

pinça, pinçaras [pinças], pincers.

pinta, v. açumbre.

pintar, to paint.

pintor, a painter.

piscado, a fish.

piscador, a fisher.

pixa, a mans yard.

plaça, a market place.

planeta, a planet.

plantar, to plant.

plata, a trencher; plato [plata], siluer.

platero, a goldsmith.

platillo, a sawcer.

plato, a platter; plata [plato], plate.

plazer, to please.

plegar, to fould.

plomo, lead.

pluma, a pen; a quill; a feather.

pobre, poore; pobre hombre, a poore man.

poco, poco a poco, softly; v. pequeño.

poderoso, mightie.

podrir, to rot.

pollería, poultrie.

pollero, a poulter.

pollo, a chickin.

por, for.

portar, to carry.

postremo, v. postrera.

postrera, postrera, postremo, the last. 
potencia, power.

potro, a colt.

pozo, v. fuente.

prado, a meadow.

precio, price.

predicar, to preach.

preguntar, to aske; to demaund.

premio, a reward.

prima, a shee cousin.

primavera, the spring.

primo, a cousin; mi primo, my cousin.

princesa, a princesse.

príncipe, a prince.

prior, a prior.

prioressa, a prioresse.

priuada, a priuie.

procurado, procured.

procurador, an attorney.

procurar, to procure.

propheta, a prophet.

prostrado, prostrated.

prostrar, to prostrate.

públicamente, púplicamente [públicamente], pupliquely.

público, púplico [público], openly.

pueblo, people.

puente, a bridge.

puerco, a hogge.

puerta, a doore; a portch.

pulga, a flea; a flee.

pulgar, the thumbe.

punçón, a bodkin.

puta, $a$ whore; v. ramera.

putañero, $a$ whoremonger.

putaria, a stewes.

quadrado, foursquare.

qual, which.

qualidad, qualitie.

quánto, how much; por quánto, for how much.

quarenta, fortie.

quartero, v. quarto.

quarto, quarto, quartero, a quarter.

quatro, foure.

que, a que, whereto; por que, wherefore;

con que, wherewith; de que, whereof.

quedar, to be with child.

quemar, to burn. querella, querel[1]a, a quarell.

querer, to will.

queso, cheese; queso fresco, curdes.

quexada, quexadas, the iawes.

quexar, to complaine.

quien, who.

quinze, fifteene.

quitar, quitar los vistidos, to put off.

rábano, rábonos [rábanos], radishes.

raer, to shaue.

rajar, to shaue.

ramera, ramera, puta, a herlot.

ramizillo, a branch.

ramo, a bough.

raposa, a foxe.

rastro, a rake.

ratonzillo, a mouse.

raýz, raýzes, rootes.

razón, reason.

recoger, recoger, ayuntar, to gather.

red, a net.

regalemente, kingly.

regaliza, licoris.

regatón, a retailer of wares.

rehazer, to do againe.

rehusar, to denie.

reír, to laugh.

reja, reja del arador, a coulter.

religión, religion.

relox, relox, horologio, a clocke.

reloxero, a clock maker.

remar, to rowe.

render, to yeeld,

reñir, renir [reñir], to chide.

reposar, to rest.

repossada, v. cama.

respondido, answered.

respuesta, an answere.

rey, a king.

reynar, to raigne.

reyno, a kingdome.

ricamente, richly.

rico, rich.

riñón, the kidney.

riqueza, riquezas, riches.

robar, to steale.

roble, an oke.

rodar, todar [rodar], to tumble. 
rodilla, a knee.

romper, rumpar [romper], to breake. romper, to breake.

rompido, broken.

ropa, a gown.

rosa, a rose.

rosal, a rose tree.

rostro, rostro, cara, a face.

roxo, redd.

rubí, a rubie.

rueca, a distaffe.

ruffián, a ruffian.

ruyseñor, the nightingale.

sábado, saturday; saterday.

sábana, sábanas, sheets.

saber, saber, conocer, to knowe.

sabiamente, wisely.

sabiduria, sabidur[i]a, wisedome.

sabiduría, sabiduría, sciencia, knowledge.

sabio, wise.

sacar, saca fuera, goe out.

sacerdote, sacerdote, clérico, a priest.

saco, a bagge.

sacudir, secudir [sacudir], to shake.

saeta, a dart.

sal, salt.

salero, a salt sellar.

salir, to goe out.

salmón, a sammon.

saltar, to salt.

saluado, bran.

saluador, our sauiour.

saluage, wilde.

sampoña, a pipe.

sanctidad, holinesse.

sancto, holy.

sangrar, to bleed.

sangre, blood.

sannar, to heale.

sarnoso, saruoso [sarnoso], scabed.

sartén, a pan to frie.

sastre, a tailor.

sayo, hit; a coate.

sciencia, v. sabiduría.

secar, to drie; secad mi camisia, drie my shirt.

seda, silke.

segador, a mower. segar, to mowe.

seguir, to followe.

segurón, an axe.

sellar, to seale.

semana, a weeke; a wecke.

sembrar, to sowe corne.

semejante, like.

seno, the bosome.

seña, a signe.

señar, to marke.

señor, a lord.

señora, a lady.

ser, to be.

serenna, serenna del mar, the mermaide.

seruieta, a napkin.

sesenta, sixtie.

setenta, seauentie.

setiembre, september.

seys, sixe.

sí, yea; sí señor, yea for footh.

siempre, alwayes.

sierra, a hill.

siete, seuen.

silla, a sadle; a chaire.

sillero, a sadler.

simiente, seede.

soberuia, pride.

sobreceja, the brow.

sol, the sunne.

solamente, onely.

sombrero, a hat.

sonar, to dreame.

soplar, to blowe.

sordo, deaffe.

sotil, craftie.

souar, to knead.

subir, to clyme; subir ar[r]iba, to goe vp.

sudar, to sweate.

suela, the sole of the foot.

sueño, a dreame.

suffrir, suffrir, padecer, to suffer.

suyo/a, his.

suziedad, filthy.

suzio, foule.

tábula, $a$ board.

tanto, por tanto, v. pero.

tarde, the evening; late; muy tarde, too late. 
tassa, a cup.

tauerna, a tauerne.

tauernero, a vintner.

taza, v. tassa.

teja, teças [tejas], tyles.

tejar, to tile.

tejero, a tyler.

tela, cloath of linnen.

temer, to be afeard.

temparano, early.

tenaza, ten[a]zas, tongs.

tenebla, darkenesse.

tenebroso, darksome.

tentar, to tempt.

terciopelo, veluet.

término, a terme.

teta, a dugge; las tetas, the dugges.

texedor, a weaner.

texer, to weane.

tía, an aunt.

tiempo, time.

tienda, a shop.

tierra, the earth.

tinta, inck.

tintar, to dye colours.

tintero, tintoreo [tintero], an inckhorne. tintorero, a dyer.

tintura, tintura del paĩo, dyeing of cloath.

tio, an vnckle.

tirar, to drawe.

toalla, v, tovalla.

toca, a coyfe.

tocar, to handle; to feele.

tocino, tussino [tocino]bacon.

todopoderoso, todo poderoso, allmightie.

tomar, to take.

tonelado, a tunne.

tonelero, a cooper.

topo, a moule.

tormentar, to rake.

torre, a tower.

torrezno, torreyno [torrezno], a gammon.

torta, a cake; a tart; tortas, a flawne.

tórtola, a turtle doue.

tosse, the cough.

tosser, to cough.

tovalla, a towell. trabajo, v. labor.

traer, to bring; traer, metar [sic], to leade.

trayción, treason.

traydor, a traytor.

tres, three.

treynta, thirtie.

treze, thirteene.

trillador, a thresher.

trillar, to thresh.

trinchete, a penknife.

trinidad, the trinitie.

tripa, tripas, the guts.

tripiés, $a$ trestle.

triste, sad.

triud, truid [triud], a treuet.

trocar, trocar, cambiar, to change.

trompeta, a trompet.

tropeçar, to stagger.

tuerto, crooked.

tuétano, the marrow.

turba, turuas [turbas], turffes.

tuyo, thine.

vaca, a cowe; vacas, kine.

vaina, $a$ sheath.

vallena, $a$ whall.

varón, a baron.

veintidós, veynte y dos, two and twentie.

veintitrés, veynte y tres, three and twentie.

veintiuno, veynte y vno, one and twentie.

vela, a sayle; v. candela.

velar, to watch.

venido, come.

venir, to come.

ventaja, aduantage.

ventana, $a$ windowe.

ventura, luck; mala ventura, ill lucke.

ver, ver antes, to foresee.

verano, the summer.

verça, verças, colewortes.

verdad, trueth.

verde, green.

verga, a yard; a rod.

vergüença, shame.

vergüençar, to shame.

veynte, twentie. 
vez, poca[s] vezes, seldome; otra vez, an other time.

viejo, olde.

vieldo, vieldo, avandor, a fan.

viento, winde.

vientre, the belly.

viergen, a vergin.

viernes, fryday.

viga, a beame.

villa, a towne.

villano, a farmer.

vina, $a$ vine.

vinagre, vinegre.

vino, wine; vino bastardo, bastard wine; vino clareto, claret wine.

violeta, violet.

virginidad, virginitie; maidenhead.

vivir, v. bibido.

vivir, v. bibir. vngüento, oyntment.

vno, one.

vntar, to salue; to annoint.

vomitar, vomitar, escupir, to spue; v. gomitar.

vua, vuas, grapes.

vuessa, vuessa merced, you.

vuestro, your.

xara, $a$ bolt.

$\mathbf{y}$, and.

yerno, a sonne in law.

yglesia, a church.

yo, $i$; yo soy, $i$ am.

yr, to goe.

zámpoña, v. sampoña.

zimborrio, zimbor[r]io, a pinacle. 